\title{
Determinação de Estruturas Tridimensionais de Proteínas em Solução por R.M.N.
}

\author{
ANJOS L. MACEDO* E B RIAN J. GOODFELLOW*
}

A caracterização estrutural detalhada de uma proteína é um passo fundamental para a compreensão da sua função biológica. Assim, a determinação de estruturas tridimensionais de proteínas tem sido objecto de estudo através da técnica de cristalografia de raios- $X$, nos casos em que é possível a obtenção de cristais, e mais recentemente, através da Ressonância Magnética Nuclear (RMN). Esta técnica tem vindo a adquirir uma grande importância, pois permite obter informação estrutural em solução, apesar de ainda estar restrita a proteínas de baixa massa molar (20$30 \mathrm{kDa}$, ou seja, aproximadamente, 200 a 300 amino ácidos).

A informação contida num espectro de RMN a uma dimensão (1D) é pouco resolvida, se pensarmos que todos os protões existentes na proteína irão entrar em ressonância numa gama relativamente pequena de frequências. Esta região situa-se na gama de desvios químicos de 0 a 10 ppm e pode ser visualizada na Figura 1. A introdução de técnicas de RMN a duas dimensões veio permitir obter informação estrutural detalhada acerca de todos estes protões numa molécula. As experiências homonucleares essenciais para a aquisiçao de dados necessários à determi-

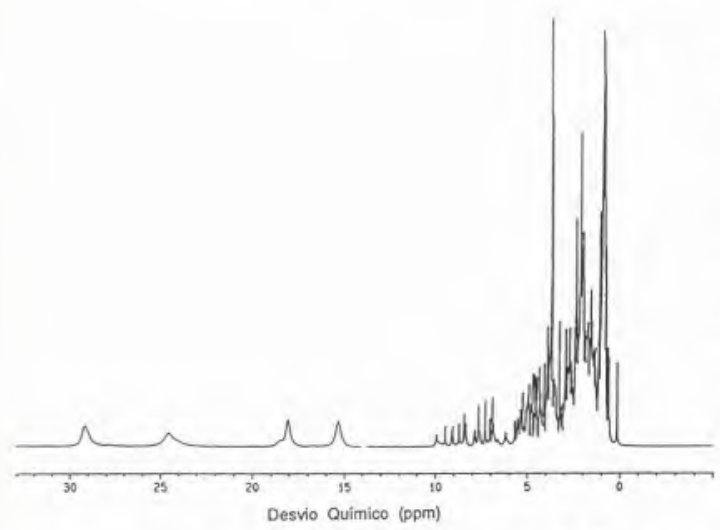

Fig. 1- Espectro de RMN de protões 1D de uma proteína contendo um centro [3Fe-4S]: Ferredoxina II isolada de bactérias redutoras de sulfato. A região diamagnética não está resolvida devido ao grande número de ressonâncias (esta proteína tem uma cadeia polipeptidica com 58 amino ácidos). Na região 10-30 ppm são detectados apenas alguns sinais bastante alargados, devidos aos protões dos ligandos do centro de ferro e enxofre (adaptado da ref. 7). nação de uma estrutura em solução são:

- $\operatorname{Cos} Y(' C o r r e l a t e d ~ S p e c t r o s-$ copy') que permite obter correlações escalares entre protões directamente acoplados, através de uma constante J.

- TOCSY ('Total Correlation Spectroscopy') permite obter correlações entre todos os protões de um amino ácido, e identificar e atribuir os sistemas de spin que os caracterizam.

NOESY ('Nuclear Overhauser Effect Spectroscopy') dá informação espacial de protões vizinhos, pois o efeito em que se baseia é proporcional ao inverso da sexta potência da distância. É essencial na determinação da estrutura.

A metodologia, que irá ser ilustrada com um caso real, passa pela aquisição e análise dos espectros bidimensionais, através de uma atribuição específica das ressonâncias nos espectros TOCSY, seguida da deteç̧ão e quantificação de sinais nos espectros NOESY, obtendo-se distâncias interprotónicas e outros constrangimentos estruturais. Com estes dados é possível determinar uma estrutura (ou uma família de estruturas) utilizando o algoritmo das 'distâncias geométricas', a qual pode ser refinada através de métodos de Dinâmica Molecular.

A presença de centros metálicos em proteínas pode alterar as propriedades destas moléculas: se o centro for paramagnético, ou seja, se o spin electrónico total for diferente de zero (devido à presença de electrões desemparelhados), a região da proteína próxima do metal é afectada, pela interacção magnética entre o spin do núcleo em ressonância e o spin electrónico. Este tipo de interacção causa grandes desvios químicos para regiões fora do envelope de ressonâncias entre 0-10 ppm e os sinais são normalmente alargados (devido ao aumento das velocidades de relaxação) (Figura 1). Estes sinais, apesar de serem mais difíceis de detectar devido à sua fraca intensidade, podem ser bastante informativos no estudo de centros activos de proteínas, pois reflectem indirectamente as varia- ções dos estados de oxidação-redução, de estrutura (por exemplo, devido a interacção com substratos), etc. No caso de proteínas contendo centros com mais de um átomo metálico, como é o caso das ferredoxinas (1) (proteínas envolvidas em processos de transferência electrónica, que contêm centros do tipo [4Fe-4S], [3Fe-4S] ou [2Fe-2S]) o estudo da dependência de temperatura dos desvios químicos dos protões $\beta-\mathrm{CH}_{2}$ das cisteínas que coordenam o centro, permitem inferir acerca das suas propriedades electrónicas e magnéticas: determinação de localização/deslocalização electrónica e modelo de acoplamento de spins no agregado $(2-6)$.

\section{DETERMINAÇÃO DE UMA ESTRUTURA POR RMN.}

\section{A desulforedoxina.}

A desulforedoxina (Dx) é uma proteína simples, purificada de bactérias redutoras de sulfato, Desulfovibrio gigas, contendo um centro metálico do tipo Fe-4S(Cisteína), semelhante ao encontrado nas rubredoxinas $(\mathrm{Rb})$ : um único ferro coordenado tetraedricamente a quatro enxofres de cisteínas da cadeia polipeptí-

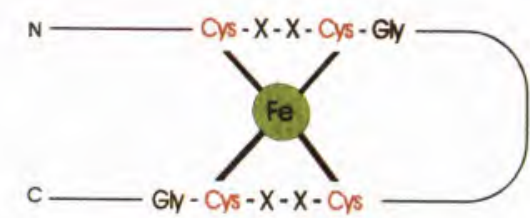

Dx

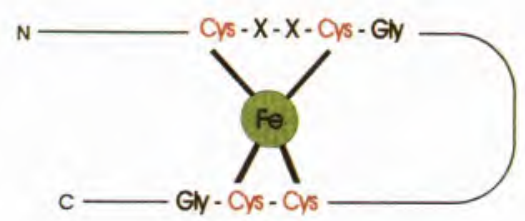

Rd

Fig. 2 - Esquema representativo da ligação dos centros de Fe à cadeia polipeptídica da Desulforedoxina (Dx) e da Rubredoxina (Rb) . Cys: cisteína; Gly: glicina. (adapatado da ref. 8 ) 
dica. Esta proteína é isolada na forma de um dímero de subunidades idênticas cada uma com cerca de 4 kDa, ou seja, 36 amino ácidos. Cada subunidade contém um centro [Fe4S], com um ião $\mathrm{Fe}^{3+}$ num estado de spin alto $(S=5 / 2)$. A esfera de coordenação da proteína apresenta diferenças geométricas relativamente à Rd, devido a diferenças no espaçamento de duas das cisteínas coordenantes $(8,9)$. Como se pode ver na Figura 2, das quatro cisteínas que coordenam o átomo de ferro na Dx duas são consecutivas, dando origem a uma geometria tetraédrica distorcida.

Como veremos mais adiante, a presença de centros paramagnéticos numa proteína pode trazer novas complicações à determinação da sua estrutura por RMN. No caso da Dx, o paramagnetismo do ferro $(\mathrm{S}=5 / 2)$ leva a que hajam alguns sinais de protões próximos do centro metálico com propriedades de relaxação muito diferentes e que não são detectados nas experiências usadas para determinação de estruturas em sistemas diamagnéticos. Uma maneira de ultrapassar este problema é a substituição do ferro por um outro metal diamagnético. Foram já determinadas estruturas tridimensionais por RMN de rubredoxinas substituídas com $\mathrm{Cd}, \mathrm{Hg}$ e $\mathrm{Zn}$ $(10,11)$.

No caso da Dx o ferro pode ser substituído usando um método simples, por $\mathrm{Ni}$, Co (12) e $\mathrm{Zn}$. A forma da Dx com $\mathrm{Zn}(\mathrm{Dx}(\mathrm{Zn}))$ é diamagnética e dá origem a sinais de RMN, que podem ser utilizados para determinar a estrutura da proteína em solução.

\section{Os sinais de RMN $1 D$ da Dx}

$\mathrm{O}$ espectro 1D da Dx no estado oxidado, Dx $\left(\mathrm{Fe}^{3+}\right.$ ) (Figura 3A), mostra o efeito do alargamento de alguns dos sinais, provocado pela presença de ferro no estado de oxidação +3 . com $\mathrm{S}=5 / 2$ (sistema $\mathrm{d}^{5}$, spin alto) $(\mathrm{x})$. Este centro pode ser reduzido quimicamente a $\mathrm{Fe}^{2+}(\mathrm{S}=2)$, apresentando então um espectro com linhas mais estreitas (Figura 3B), devido à dimi-

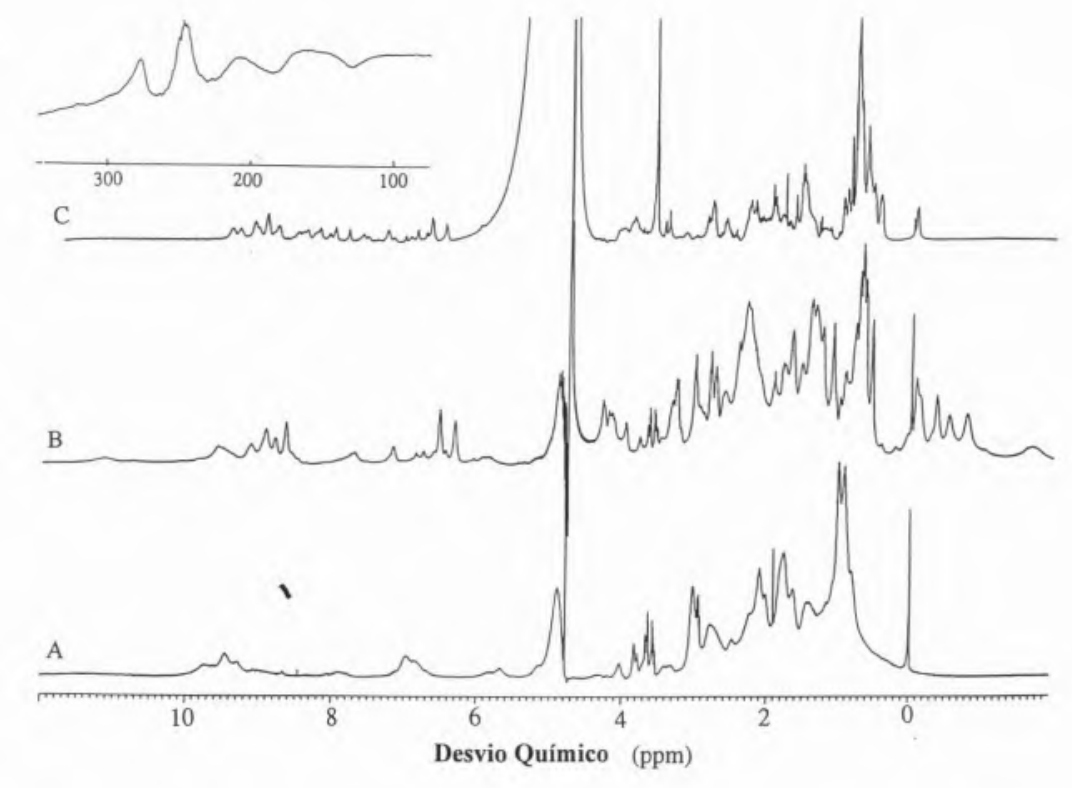

Fig. 3 - Espectros de RMN ID: forma oxidada (A) e forma reduzida (B) da desulforedoxina, Dx(Fe); (C) desulforedoxina substituída com zinco, Dx(Zn). A expansão mostra a região 100-300 ppm da Dx(Fe) na forma reduzida. (adaptado da ref. 14)

nuição do paramagnetismo. No entanto, alguns sinais têm desvios químicos a campo baixo, na região de 100-300 ppm, muito longe da região diamagnética (0-10ppm) (Figura 3C). Estas ressonâncias tão desviadas são devidas à interacção dos protões $\beta$ $\mathrm{CH}_{2}$ das cisteínas coordenadas, com o spin electrónico do ferro (13).

$O$ espectro 1D da forma $\mathrm{Dx}(\mathrm{Zn})$ (Figura 3C) mostra que todos os sinais são estreitos e se encontram na região 0-10 ppm. Neste caso, é possível fazer-se a atribuição sequencial pelos métodos 'clássicos' de RMN 2D e determinar a estrutura da proteína em solução (14).

Requisitos para a determinação de uma estrutura

Para as experiências de RMN é necessário uma proteína que seja estável durante vários dias, solúvel em água, em concentrações $>1 \mathrm{mM}$, num volume total de $\sim 500 \mu \mathrm{l}$. A sequência primária da proteína tem que ser previamente conhecida. Usando os métodos de RMN homonuclear só é possível determinar estruturas de proteínas com menos de
100 amino ácidos. Para proteínas maiores é necessário marcar isotopicamente a molécula com ${ }^{15} \mathrm{~N}$ e ${ }^{13} \mathrm{C}$ e utilizar técnicas de RMN heteronucleares (15). Este processo é bastante dispendioso, pois é necessário crescer as bactérias de onde as proteínas são isoladas, em meios enriquecidos com os isótopos respectivos, de maneira a que estes sejam incorporados nas proteínas.

A aquisição dos espectros de RMN tem que ser feita em amostras da proteína em solução $100 \% \mathrm{D}_{2} \mathrm{O}^{*}$, e em solução $90 \% \mathrm{H}_{2} \mathrm{O} / 10 \% \mathrm{D}_{2} \mathrm{O}$. Os espectros em $\mathrm{D}_{2} \mathrm{O}$ permitem complementar a informação obtida nos espectros em $\mathrm{H}_{2} \mathrm{O}$, relativamente aos sinais que aparecem sobrepostos com o sinal da $\mathrm{H}_{2} \mathrm{O}$.

* A água da solução é substituída por água deuterada e todos os protōes que permutam com $o$ solvente deixam de ter sinal no espectro de NMR de protões. logia

Determinação da estrutura. Metodo-

A determinação de uma estrutura proteica pela técnica de RMN 


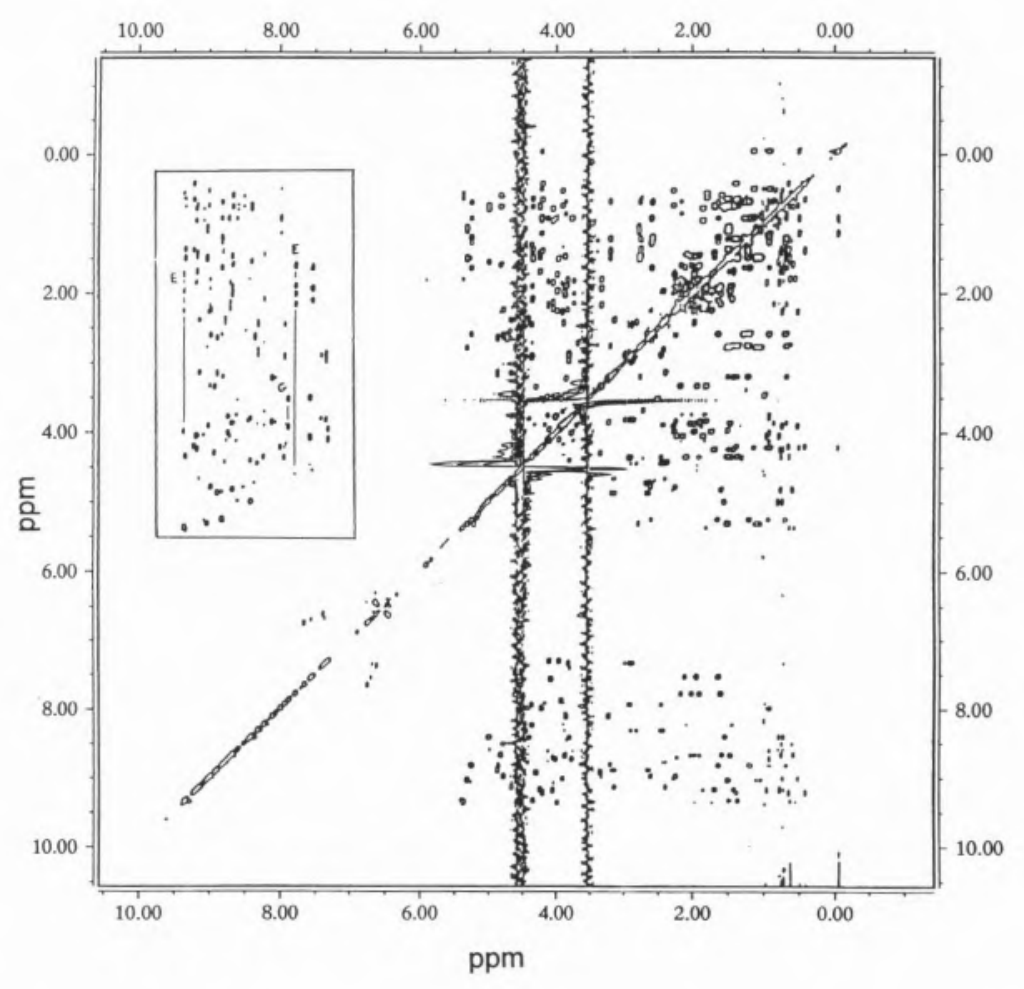

Fig. 4 - O espectro TOCSY da Dx(Zn). A caixa delimita a região onde aparecem as correlações entre os protões $\mathrm{NH}$ e o resto dos protões do mesmo amino ácido (adapatado da ref. 14). homonuclear pode ser sistematizada em várias etapas, que são apresentadas de seguida.

i) A identificação dos sistemas de spin é feita através das experiências TOCSY e COSY. Na Figura 4 é apresentado um espectro TOCSY da $\mathrm{Dx}(\mathrm{Zn})$, onde se mostram as correlações entre os protões $\mathrm{NH}$ da ligação peptídica, e todos os protões do mesmo amino ácido (sistema de spin). Desta maneira é possível fazer um agrupamento de todos os sistemas spin devido ao tipo de padrão de picos cruzados. Por exemplo, na Figura 5 são apresentados em forma de esquema os espectros TOCSY 2D esperados para os sistemas de spin dos amino ácidos cisteína, C, valina, V, e tirosina, $\mathrm{Y}$.

O espectro COSY permite esclarecer a atribuição dos sistemas de spin, em casos de sobreposição de ressonâncias, no espectro TOCSY.

ii) Numa segunda fase, é necessário estabelecer correlações entre cada um dos sistemas de spin. O espectro NOESY a duas dimensões
A EXPERIÊNCIA DE RMN ID. A técnica de ressonância magnética nuclear de protões (núcleos $1 \mathrm{H}$ ) baseia-se na interação entre um campo magnético oscilante, produzido por ondas de frequência de rádio, e a magnetização global da amostra. Esta magnetização é devida à orientação dos spins nucleares, $\mathrm{I}=1 / 2$, num campo magnético estático, ao qual a amostra é sujeita, e resulta da soma das populações com momento magnético de spin nuclear $\mathrm{m}_{\mathrm{I}}=$ $1 / 2$ e $m_{I}=-1 / 2$ (dadas pela distribuição de Boltzmann). Segundo o esquema 1 , a magnetização, $M$, que se encontra alinhada segundo o eixo dos zz e é paralela ao campo magnético aplicado, ao interactuar com uma onda de frequências de rádio, RF (campo electromagnético aplicado perpendicularmente a este eixo, $\mathrm{B}_{1}$ ) e com uma duração definida (impulso de $90^{\circ}$ ), vai passar a en- contrar-se no plano xy. O sinal adquirido é o resultante de todas as ondas detectadas para cada um dos núcleos individuais, que relaxam para a sua posição inicial, através de interacções com os núcleos vizinhos. Este sinal tem o nome de FID (do inglês 'free induction decay'). Depois de um tempo de espera, $t R$, suficientemente longo para que o sistema volte às condições iniciais, é possível repetir esta sequência de maneira a acumular a informação e melhorar a razão sinal/ruído do espectro final. A utilização da operação matemática transformada de Fourier, FT, permite individualizar cada uma das ondas do FID, que decaem com frequências diferentes, e passar de uma coordenada temporal para uma coordenada de frequências, obtendo-se assim o espectro de RMN a uma dimensão, ID (esquema 2).
Esquema 1. O impulso de $90^{\circ}$. Interacção de uma onda de frequências de rádio (RF) com a magnetização global da amostra (M) (adaptado da ref. Derome, A.E., 1987)

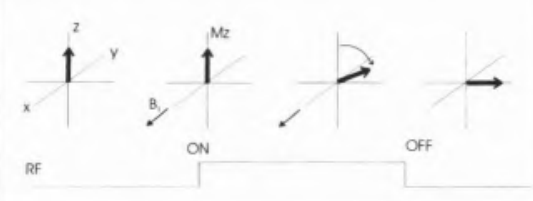

Esquema 2. Sequência de impulsos numa experiência de RMN a uma dimensão.

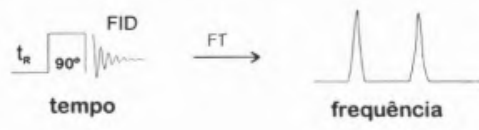


contém informação sobre a proximidade dos protões dentro de um sistema de spin e entre diferentes sistemas de spin (ver Figura 6). O aparecimento de um destes sinais de correlação entre um par de protões, significa que eles se encontram a uma distância inferior a $5 \AA$.

Por comparação dos espectros TOCSY e NOESY é possível fazer-se uma atribuição sequencial dos sistemas de spin a qual é seguida de uma atribuição específica a cada um dos amino ácidos, por comparação com a sequência primária da proteína ( $\mathrm{Fi}$ gura 7). Por exemplo, se conseguirmos detectar correlações entre dois sistemas de spin do tipo C V e encontrarmos só uma combinação deste tipo na sequência primária, pode fazer-se a atribuição específica imediata ...C21-V22.... Se houver mais do que uma combinação CV, é necessário identificar novas correlações entre CV e o sistema de spin seguinte, e identificar na sequência a nova combinação de três sistemas de spin.

Outro tipo de informação estru- tural obtida para a Dx(Zn) através dos resultados de RMN, é que esta tem, em solução, uma conformação dimérica totalmente simétrica. Esta conclusão baseou-se no facto de o número de ressonâncias de protões $\mathrm{NH}$ detectadas ser igual ao número de amino ácidos da proteína. Além disso, nos espectros obtidos em $\mathrm{D}_{2} \mathrm{O}$, a presença dos protões $\mathrm{NH}$ da cadeia principal, indica que estes se encontram protegidos do solvente, estando, normalmente, envolvidos em ligações do tipo 'pontes de hidrogénio'.

iii) A estrutura secundária. Usando a informação recolhida no espectro NOESY, podemos inferir acerca do tipo de elementos estruturais secundários na estrutura da proteína. A Figura 8 apresenta os resultados obtidos para a Dx $(\mathrm{Zn})$.

A presença de sinais intensos do tipo NH-H $\alpha(\mathrm{i}, \mathrm{i}-1)$ e de sinais fra$\cos \mathrm{NH}-\mathrm{H} \alpha(\mathrm{i}, \mathrm{i})$ detectados para os resíduos dos segmentos $\mathrm{V} 6$ a $\mathrm{C} 9 \mathrm{e}$ V16 a L19, definem uma conformação 'estendida'. Os sinais intensos
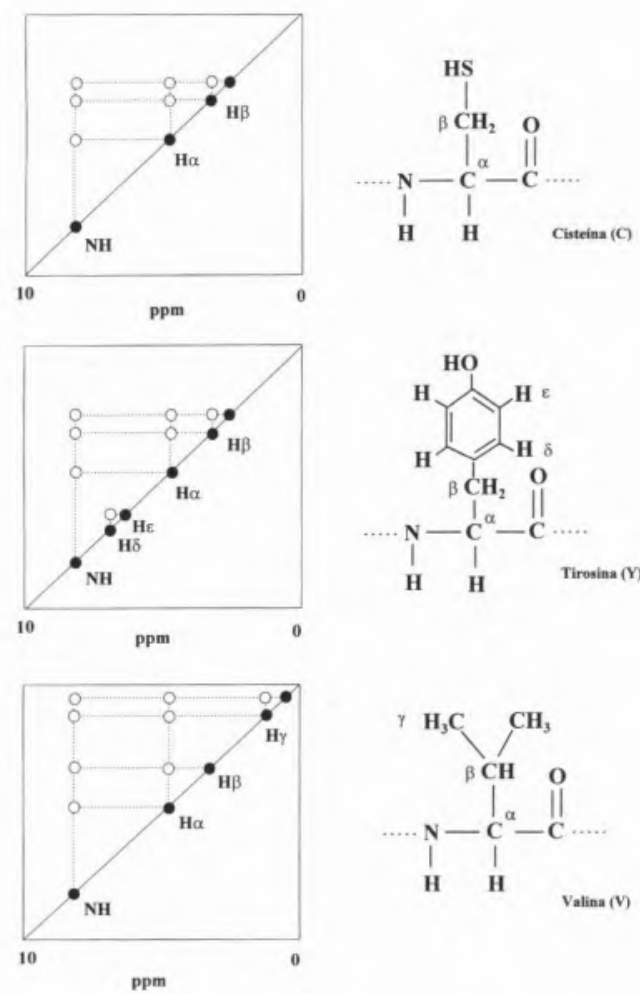

Fig. 5 - Esquema representativo dos picos cruzados esperados num espectro TOCSY, para os seguintes sistemas de spin: cisteína, C, valina, V, e tirosina, Y.
A EXPERIÊNCIA DE RMN $2 D$. Num espectro COSY (correlação linear dos desvios químicos) pretende-se obter correlações entre núcleos $1 \mathrm{H}$ directamente acoplados (< 3 ligações químicas). Este efeito obtem-se aplicando a seguinte sequência de impulsos:

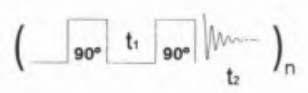

Numa primeira fase de preparação do sistema, o impulso de $90^{\circ}$ cria uma magnetização no plano xy. Durante o tempo $\mathrm{tl}$ as várias componentes da magnetização evoluem por interacção com o ambiente químico. O segundo impulso de $90^{\circ}$ provoca a transferência de magnetização para os outros spins nucleares acoplados, sendo por isso considerado como um impul- so de mistura. É esta etapa caracteriza as experiências de 2D. A aquisição do FID faz-se imediatamente a seguir ao segundo impulso e é definida como um período de detecção, sendo função de $t 2$. Esta sequência é repetida $\underline{n}$ vezes, para um conjunto de valores de tl incrementados de $\Delta$ t (para melhorar a razão sinal ruído são acumulados vários transientes para cada valor de t1). O processamento dos dados obtidos passa pela aplicação de uma transformada de Fourier em relação a t2, gerando $\underline{\mathrm{n}}$ espectros numa coordenada de frequências definida como f2. A segunda transformada de Fourier é agora aplicada na direç̧ão temporal tl (pois os sinais individuais de cada um dos espectros na direcção f2 podem agora ser modulados, devido às diferenças nas suas intensidade e/ou nas suas fases, como reflexo da variação periódica duma propriedade do sistema) dando origem à segunda dimensão, fl. O resultado é uma intensidade espectral em função de duas frequências, $\mathrm{fl}$ e f2, que apresenta na diagonal picos correspondentes ao espectro 1D, ou seja, as componentes da magnetização com frequências iguais durante t 1 e t2. Os sinais fora da diagonal (picos cruzados) correlacionam dois núcleos acoplados, por exemplo, A e $X$, tendo coordenadas $(\delta \mathrm{A}, \delta \mathrm{X})$ e $(\delta \mathrm{X}$, $\delta A)$.

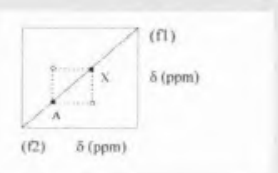




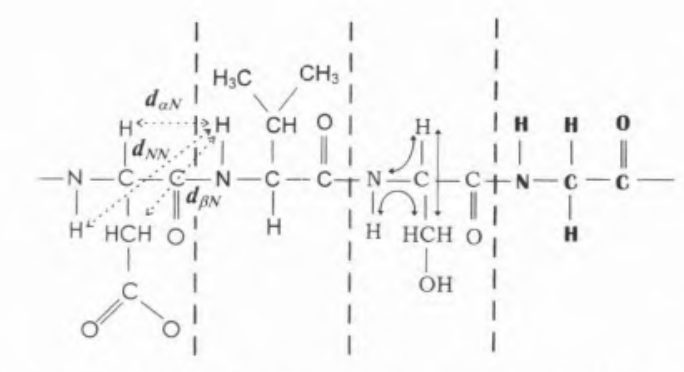

$-\operatorname{Asp}(\mathrm{D})-\operatorname{Val}(\mathrm{V})-\operatorname{Ser}(\mathrm{S})-\operatorname{Gly}(\mathbf{G})$

Fig. 6 - Sequência de amino ácidos -D-S-V-GAs setas a tracejado e a negro representam, respectivamente, os NOE's sequências (entre protões de dois amino ácidos consecutivos) e a negro as correlações TOCSY e/ou NOESY entre os protões do mesmo amino ácido.

$\mathrm{NH}-\mathrm{NH} \alpha(\mathrm{i}, \mathrm{i}+1)$ para E10 a G13, em conjunto com o padrão $\mathrm{NH}-\mathrm{H} \alpha(\mathrm{i}, \mathrm{i}-$ 1) é indicativo se temos uma estrutura folha- $\beta$, curva e folha- $\beta$, nesta região da proteína (Figura 9). Servem neste caso como confirmação os NOEs de 'longa distância' do tipo NH-NH entre Y7-Vl6 e C9-Q14; $\mathrm{H} \alpha$-H $\alpha$ entre V6-K17 e K8-V15; NH-H $\beta$ entre Q14-C9, G13-C9 e D5-V18; NH-H $\gamma, \delta$ entre D 5-V 18. Além disso, os resultados obtidos nos espectros em $\mathrm{D}_{2} \mathrm{O}$ sugerem a presença de protões com velocidades de permuta lentas (protões pouco expostos ao solvente), ou seja, possíveis ligações de hidrogénio entre os protões dos resíduos da estrutura folha- $\beta$ desta região da proteína.

Os resíduos E20 a V27 têm poucos sinais de NOE, o que indica uma estrutura em folha $\beta$. O padrão dos NOEs perto de C-terminal é indicativo duma curva em C29, G30. O arranjo global da cadeia polipeptídica tem que deixar o C-terminal na proximidade dos resíduos D5-K8, pois são visíveis NOEs entre V6 e K35, e K8 e K35.

iv) Em geral para se determinar uma estrutura é necessário fazer um estudo quantitativo. Cada sinal cruzado, num espectro NOESY, tem um determinado volume, que é propor- cional à distância entre os protões que geram esse sinal.

O cálculo dos volumes é feito através da integração dos sinais. A calibração é feita utilizando o volume de um sinal cuja distância correspondente seja conhecida como, por exemplo, as distâncias fixas entre protões $H \beta$. Este processo dá uma lista de distâncias entre os vários protões na proteína.

Os espectros em $\mathrm{D}_{2} \mathrm{O}$ podem dar informação acerca das ressonâncias que aparecem sobrepostas com o pico da $\mathrm{H}_{2} \mathrm{O}$. Além disso, também podem ser utilizadas as ressonâncias dos protões NH que estão em situação de permuta lenta, para obter restrições de distâncias NH-O se, depois de uma primeira determinação de uma família de estruturas, os protões $\mathrm{NH}$ se encontrarem próximos de um oxigénio carbonilo. v) Utilizando as distâncias determinadas e tendo em conta a sequência primária da proteína, é possível calcular a estrutura, ou melhor, uma família de estruturas, pois normalmente há mais do que uma estrutura que satisfaz a lista de condições impostas pelo cálculo das distâncias interprotónicas. Assim, quanto maior for o número de condições, mais precisa é a família de estruturas obtida, o que se reflecte no parâmetro 'raíz quadrada da média do quadrado dos desvios' (em inglês "root mean square deviation", RMSD) dos protões da cadeia principal da proteína, através de valores inferiores a $1 \AA$.

Os cálculos necessários para gerar estas estruturas utilizam um algoritmo complexo e é necessário um computador com grande capacidade de cálculo.

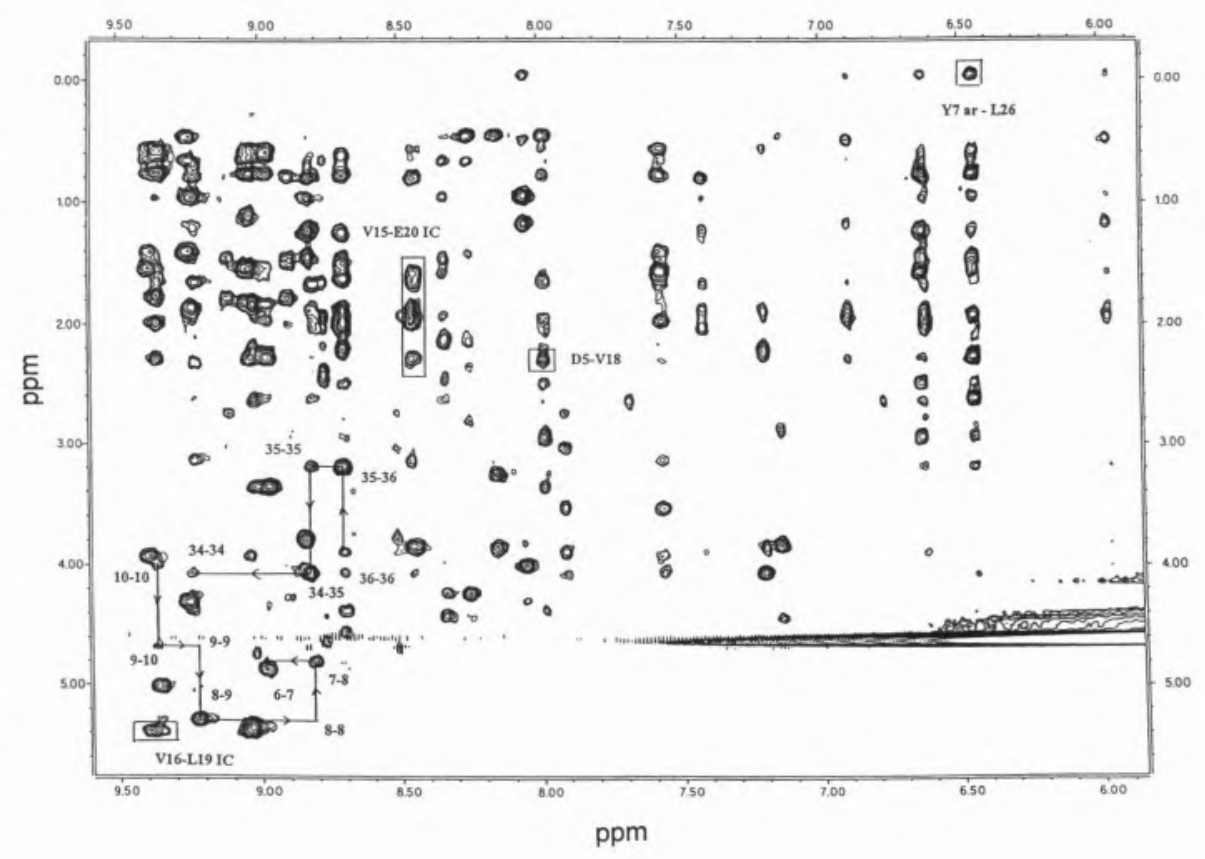

Fig. 7 - Espectro NOESY da Dx(Zn), mostrando as correlações entre sistemas de spin e a correspondente atribuição específica. IC: correlações entre os monómeros (adaptado da ref. 14). 


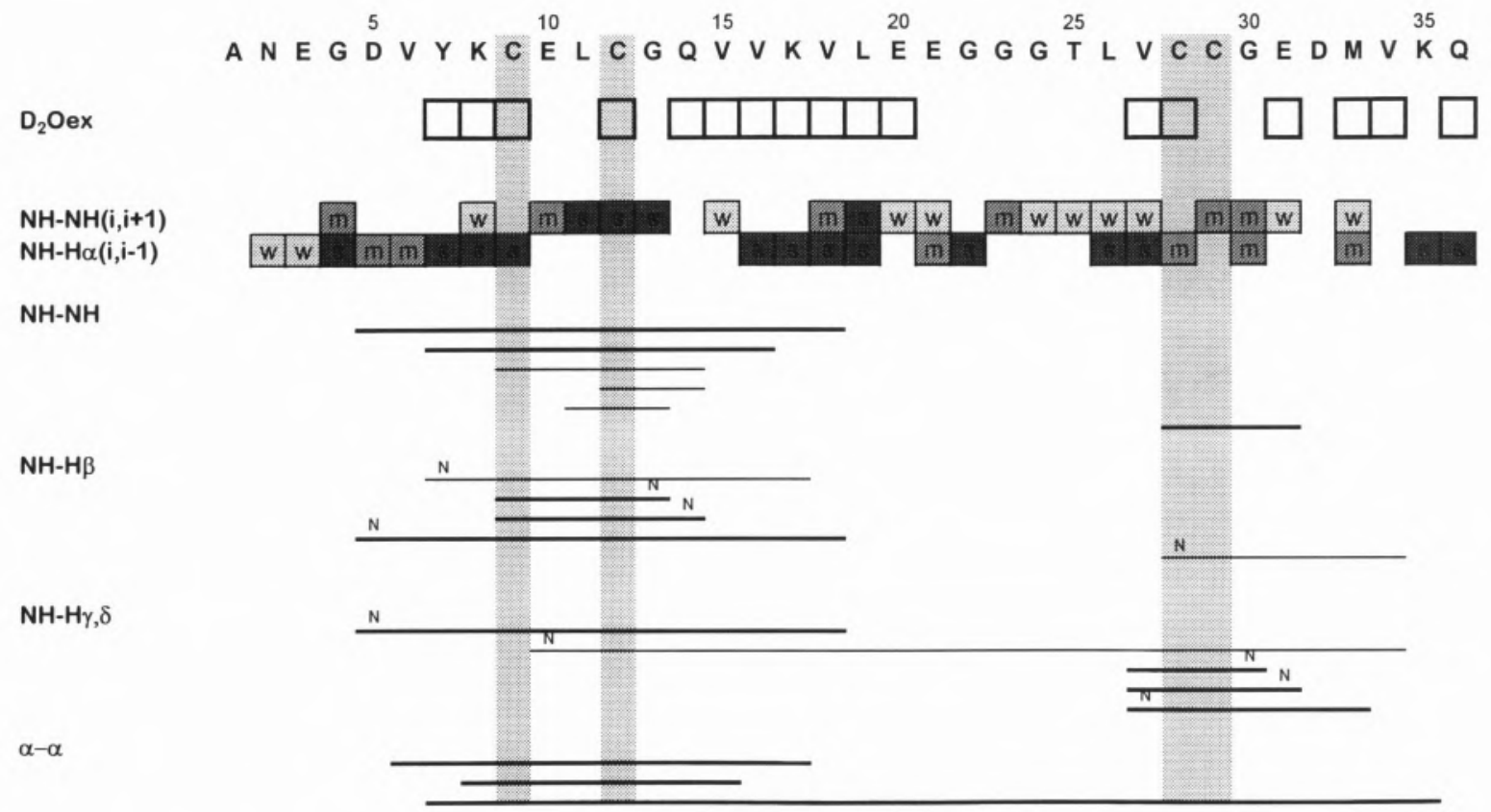

Fig. 8 - Representação esquemática dos protões $\mathrm{NH}$ com velocidade de permuta lenta ( $\mathrm{D}_{2} \mathrm{Oex}$ ), que são representados por caixas; dos $\mathrm{NOE}$ 's sequenciais, $\mathrm{NH}$ - $\mathrm{NH}(\mathrm{i}, \mathrm{i}+1)$ e $\mathrm{NH}-\mathrm{H} \alpha(\mathrm{i}, \mathrm{i}-1)$ (entre protões de dois amino ácidos vizinhos), e de alguns NOEs de 'longa distância', NH-NH (entre protões de amino ácidos não sequenciais). As letras s, m e $w$, representam as intensidades dos picos cruzados, respectivamente, forte, médio e fraco. A intensidade das linhas corresponde à intensidade dos picos cruzados (adaptado da ref. 14).

No caso da Dx(Zn) foi utilizado um programa de "distâncias geométricas" (nome dado pelo algoritmo utilizado) chamado DIANA, que permite gerar uma família de estruturas, através dos resultados obtidos por tratamento dos espectros de RMN.

O RMSD calculado para a famíla de estruturas da Dx foi $0.63 \AA$. Mas os valores de RMSD para cada resíduo variam ao longo da cadeia principal indicando uma desordem estrutural. A região E20-T25 e o Nterminal têm os valores mais altos de RMSD. A Figura 10 ilustra a sobreposição das 17 estruturas com energia mínima (utilizando o programa 'DIANA') obtidas para a Dx(Zn), onde é possível ver a desordem apresentada pela região E20T25.

\section{SISTEMAS PARAMAGNÉTICOS}

As técnicas de RMN bidimensional só foram muito recentemente aplicadas ao estudo de sistemas para- magnéticos. Estes sistemas caracterizam-se por grandes velocidades de relaxação e grandes janelas espectrais. Assim, ao aplicar estas técnicas nas condições usuais, não é possível detectar os sinais dos protões dos resíduos da cadeia polipeptídica que interactuam com o centro paramagnético.
No entanto, podem utilizar-se as mesmas experiências 2D de COSY e NOESY, mas tendo em conta que a sequência de impulsos deverá ser muito mais rápida, com tempos de mistura bastante curtos, de maneira a não se perder a informação acerca destas ressonâncias. Para isso é necessário fazer experiências em que os

Fig. 9 - Esquema representativo da estrutura folha beta, num arranjo antiparalelo da cadeia principal. As pontes de hidrogénio estão indicadas por traços paralelos e as setas representam as interacções $\mathrm{NH}-\mathrm{H} \alpha(\mathrm{i}, \mathrm{i}-1), \mathrm{NH}-\mathrm{H} \alpha(\mathrm{i}, \mathrm{i})$ e $\mathrm{H} \alpha-\mathrm{H} \alpha$ 

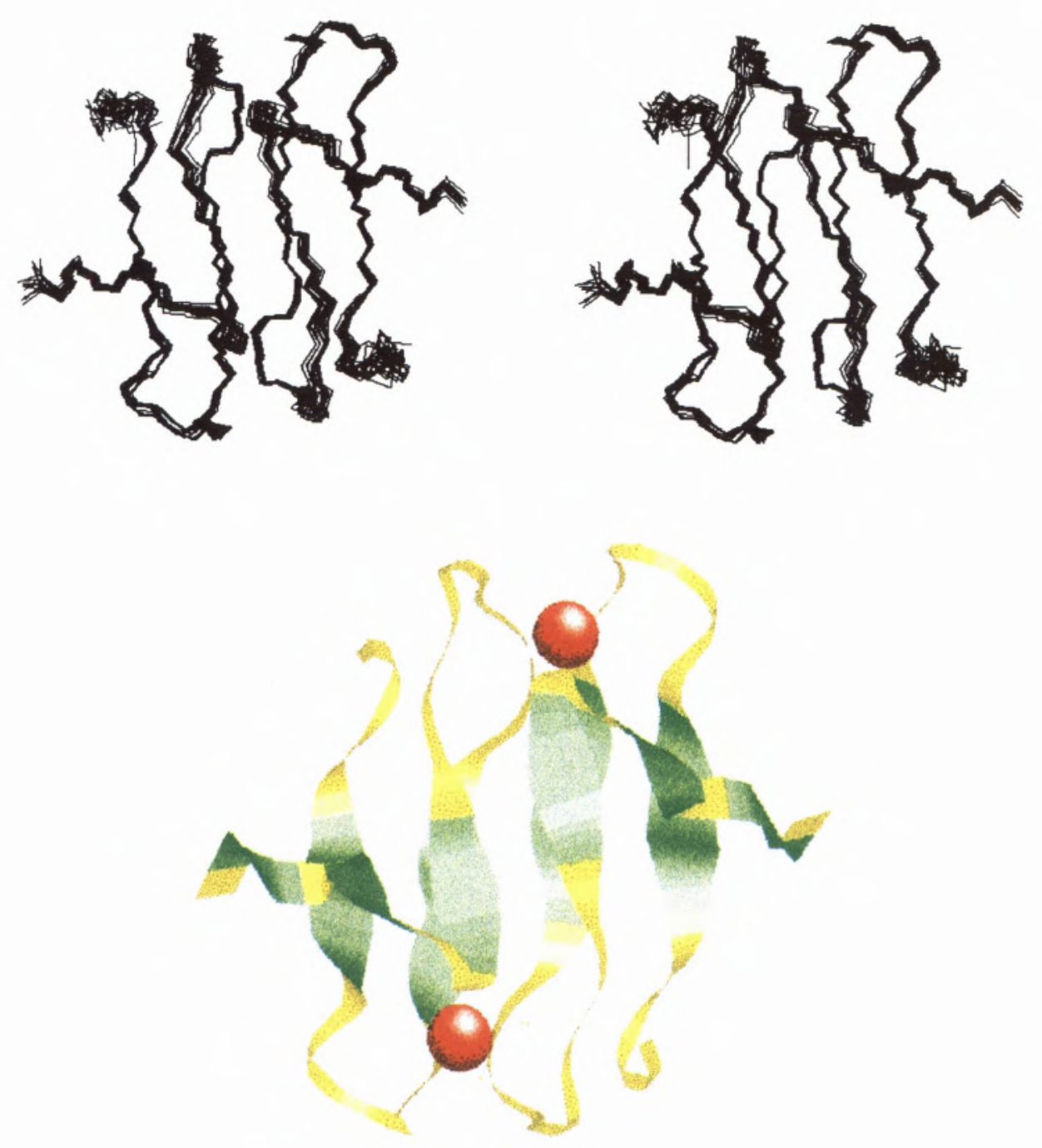

Fig. 10 - Representação em estereo das 17 estruturas da Dx(Zn) obtidas por RMN de protões. Estas estruturas representam uma família com uma energia mínima, cujo RMSD é de 0.59 Å. A cores está representada a estrutura de cristalografia de raios-X (17) da mesma molécula (RMSD entre a familia de RMN e a estrutura de Raios-X igual 1.00Å) (adaptado da ref 14).

tempos de espera entre os impulsos sejam da ordem de grandeza do inverso da largura dos sinais a detectar. Neste caso, porém, perde-se informação acerca da região diamagnética da proteína (Figura 11). Assim, na determinação da estrutura em solução deste tipo de sistemas, é necessário realizar experiências separadas (com diferentes condições) para as duas regiões e depois tentar obter informação que as correlacione.

A metodologia a seguir deve ter em conta que:

i) Os núcleos que se encontram afastados do centro paramagnético não são afectados pela sua presença, o que quer dizer que as correlações entre eles podem ser detectadas pelos métodos de rotina em RMN multidimensional.

ii) Os núcleos que se encontram numa esfera de aproximadamente $8 \AA$, relativamente ao centro metálico, são normalmente afectados pelo acoplamento com electrões desem- parelhados e dão origem a sinais alargados que se encontram fora do envelope diamagnético. Neste caso, as experiências de RMN devem ser feitas com tempos de aquisição e mistura curtos, de modo a que as correlações entre protões que relaxam rapidamente possam ser detectadas.

iii) A maior dificuldade reside na detecção de sinais que, apesar de sentirem o paramagnetismo do cen- 


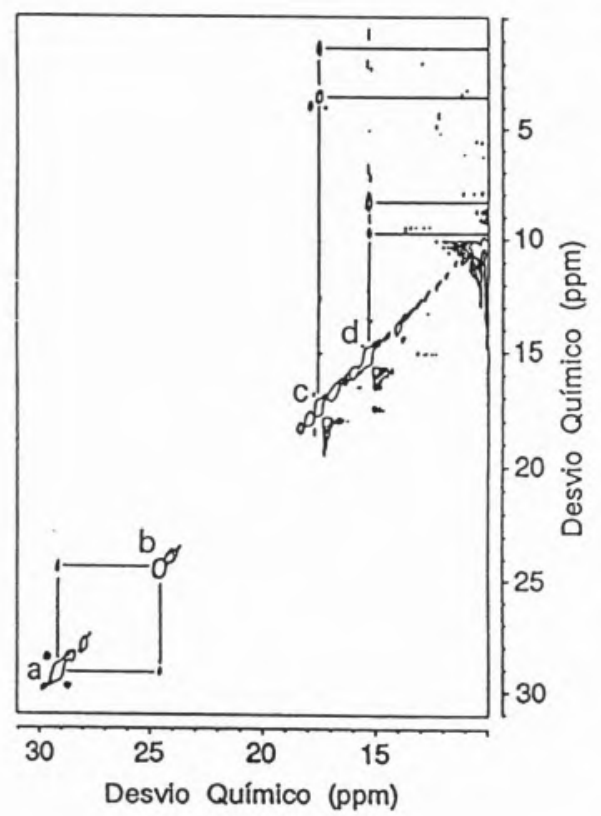

Fig. 11- Espectro de NOESY da Ferredoxina II isolada de Desulfovibrio gigas, adquirido com um tempo de mistura de $2 \mathrm{~ms}$. Apenas se representa a região a campo baixo, característica das ressonâncias paramagnéticas, pois nestas condições não é possivel obter informação acerca da região diamagnética (adaptado da ref. 7).

tro, não se encontram desviados do envelope diamagnético. As correlações entre estes sinais são difíceis de obter, devido às suas propriedades e para detectá-los na região diamagnética (com grande densidade de picos cruzados), é necessário jogar com as diferenças nas propriedades de relaxação.

iv) As correlações entre sinais com relaxação rápida e sinais com relaxação lenta (região paramagnética / região diamagnética) podem ser determinadas através de experiências NOESY, com tempos de mistura (vide caixa) próximos do inverso da média das velocidades de relaxação dos dois tipos de sinais, e de experiências de NOE, a uma dimensão (NOE 1D).

$\mathrm{Na}$ figura 12 estão resumidas as técnicas de RMN utilizadas na determinação de estruturas de proteínas paramagnéticas, assim como o tipo de informação obtida.

\section{AGRADECIMENTOS.}

Agradecemos a todos os colegas do grupo de Bioinorgânica e de Bioquímica Física de Proteínas, na FCTUNL e do Departamento de Química da Univ. de Aveiro, que estiveram envolvidos neste trabalho e contribuiram para a realização deste artigo. À Prof. I. Moura (UNL), Prof. J.J.G. Moura (UNL) e Prof. J. Pedrosa (UA) gostaríamos de agradecer o seu constante envolvimento e empenhamento na nossa actividade científica, o que nos permitiu obter o 'background' necessário à escrita deste artigo.

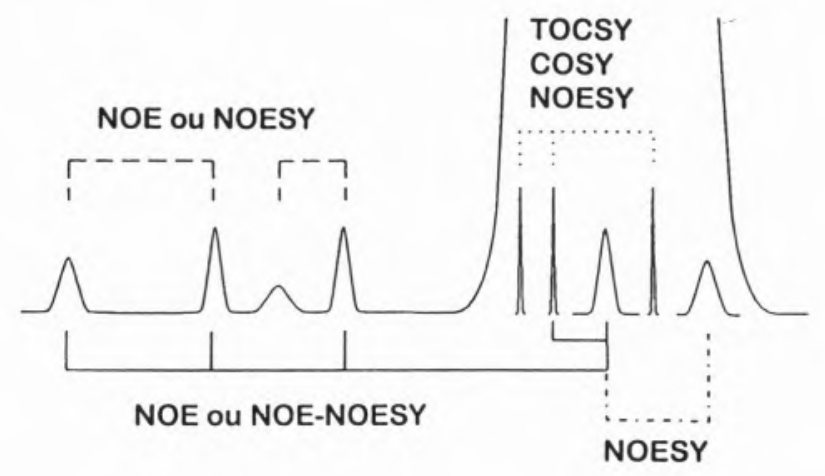

Fig. 12 - Esquema de espectro de RMN 1D para uma proteína paramagnética e experiências a realizar para obter correlações entre os vários tipos de sinais (adaptado da ref.16)
* Departamento de Química e Centro de Química Fina e Biotecnologia, Faculdade de Ciências e Tecnologia, Universidade Nova de Lisboa, 2825 Monte de Caparica

et Departamento de Química, Universidade de Aveiro, 3810 Aveiro

\section{BIBLIOGRAFIA}

\section{Referências gerais}

Bertini, I., Molinari, H., Niccolai, N. (1991) NMR and Biomolecular Structure, VCH, Weinheim, cap. 5.

Derome, A.E. (1987) Modern NMR Techniques for Chemistry Research, Pergamon Press, Oxford.

Gil, V.M.S., Geraldes, C.F.G.C. (1987) Ressonância Magnética Nuclear. Fundamentos, Métodos e Aplicações, Fundação Calouste Gulbenkian, Lisboa.

Redfield, C. (1993) NMR of Macromolecules. A Practical Approach, Roberts, G.C.K., ed., IRL Press, Oxford, cap. 4.

Wüthrich, K. (1986) NMR of Proteins and Nucleic Acids, Wiley, New York.

\section{Referências especificas}

1. Moura, IJ.G., Macedo, A.L., Palma, P.N. (1994) Methods in Enzymology. Inorganic Microbial Sulfur Metabolism., Peck, H.D., Ir., LeGall, J., eds., Academic Press, Inc., 243, cap. 12

2. Salmeen, I., Palmer, G. (1972) Arch. Biochem. Biophys, 150, $767-773$

3. Dunham, W.R., Palmer, G., Sands, R.H., Bearden, A.I. (1971) Biochim.. Biophys. Acta 253, 373-384

4. Bertini, I., Briganti, F., Luchinat, C., Scozzafava, A., Sola, M. (1991) 113, 1237-1245.

5. Banci, L., Bertini, I., Briganti, F., Scozzafava, A. Oliver, M.V. (1991) Inorg. Chim. Acta 180, 171-175.

6. Macedo, A. L., Moura, I., Moura, J.J.G., LeGall, J., Huynh, B.H. (1993) Inorg. Chem., 32, 1101-1105.

7. Macedo, A. L., Palma, P. N., Moura, I., LeGall, I., Wray, V., Moura, J.J.G. (1993) Magn. Res. Chem., 31, S59-S67.

8. Bruschi, M., Moura, I., Sieker, L. LeGall, I, and Xavier, A.V. (1979) Biochem. Biophys. Res Commun. 90, 596-605.

9. Moura, I., Xavier, A.V., Cammack, R. and Bruschi, M. (1979) Biochim. Biophys.Acta 533, 156-162.

10. Blake, P.R., Park, J-B., Zhou, Z.H., Hare, D.R., Adams, M.W.W., and Summers, M.F. (1992) Protein Sci. 1, 1508-1521.

11. Blake, P.R., Park, J-B., Bryant, F.O., Aono, S., Magnuson, I.K., Eccleston, E., Howard, J.B., Summers, M.F. \& Adams, M.W.W. (1991) Biochem. 30, 10885-10895

12. Moura, I.J.G., Goodfellow, B.J., Romão, M.J., Rusnak, F., and Moura, I. (1996) Comments on Inorg. Chem., in press.

13. Xia, B. Westler, W.M. Cheng, H., Mever, I., Moulis, I. M. and Markley, J.M. (1995) J. Am. Chem. Soc. 117, 5347-50.

14. Goodfellow, B.J., Tavares, P., Romāo, M.J., Czaja, C., Rusnak, F., LeGall, J., Moura, I., and Moura, J.J.G. (1996) J. Biol. Inorg. Chem., 1, 341-53.

15. Markley, J.L. Kainosho, M. (1993) NMR of Macromole. cules. A Practical Approach, Roberts, G.C.K., ed., IRL Press, Oxford, cap. 5

16. Banci, L., Pierattelli, R. (1995) Nuclear Magnetic Resonance of Paramagnetic Macromolecules, La Mar, G.N., ed., NATO ASI serie C, Kluwer Academic Publishers, 457, 281-296.

17. Archer, M., Huber, R., Tavares, P., Moura, I., Moura, J.G. Carrondo, M.A., Sieker, L.C, LeGall, I, and Romäo, M.I. (1995) J. Mol. Biol. 251, 690-702. 